\title{
Survey on Driver's Drowsiness Detection System
}

\author{
Omkar \\ Dharmadhikari \\ SPPU, Pune
}

\author{
Revati Bhor \\ SPPU, Pune
}

\author{
Pranjal Mahajan \\ SPPU, Pune
}

\author{
H.V. Kumbhar \\ SPPU, Pune
}

\begin{abstract}
There is much attentional impairment while driving that affect driver's reaction. Among which driving while drowsy is one of the major causes behind road accidents, and exposes the driver to a much higher crash risk compared to driving while alert. Therefore, the use of an assistive system that monitor a driver's level of vigilance and alert the driver in case of drowsiness can be significant in the prevention of accidents. This paper introduces a new approach towards detection of driver's drowsiness based on yawning measurement and head movement. This involves several steps including the real time detection and tracking of driver's face, detection and tracking of the mouth contour, the detection of yawning based on measuring both the rate and the amount of changes in the mouth contour area and head movement tracking. Test results demonstrate that the proposed system can efficiently measure the aforementioned parameters and detect driver's drowsiness.
\end{abstract}

\section{Keywords}

Active contour model, Drowsiness detection, Yawn detection, Face detection, Head movement detection

\section{INTRODUCTION}

Driving while drowsy is a contributing factor to 22 to 24 percent of car crashes according to the National Highway Traffic Safety Administration (NHTSA). The car accidents caused by fatigue drivers is four to six times higher than that of near-crash or crash risk relative to alert drivers [1] as fatigue drivers fail to take correct actions prior to a collision. Ontario Ministry of Transportation's Driver's Handbook also mentions that "Drowsiness is a major fact of growing number of collisions resulting in injury and fatality. This is mainly due to the fact that driver fatigue impacts the alertness and response time of the driver thus increases the chances of getting involved in car accidents. Drowsy drivers may fall asleep at the wheel or tend to make serious, sometimes fatal driving errors. Drowsiness and fatigue can often affect driver's ability long before they notice that they are getting tired. Studies have shown that collisions involving drowsiness tend to occur during late night/early morning hours (between 2:00 a.m. and 6:00 a.m.) or late afternoon (between 2:00 p.m. and 4:00 p.m.). Studies also indicate that shift workers, people with undiagnosed or untreated sleep disorders, and commercial vehicle operators, are at greater risk for such collisions." An important irony of driver drowsiness is that the driver may be too tired to realize his own level of inattention. Hence, driver monitoring systems which can detect lack of attention and alert the driver will play an important role in the automation system of future vehicles to prevent accidents and save lives.

Different signs and body gestures can be monitored as indicators of driver fatigue. They include daydreaming while on the road, driving over the center line, yawning, feeling impatient, heavy eyes and slow reaction. A number of related works in the field have addressed the problem of detecting driver drowsiness based on the expressions of the driver's face (i.e. eye motion, yawning etc.), that will be briefly reviewed in the related work section. This paper focuses on yawning and head movement as a sign of fatigue. Unlike existing systems that suffer from computational complexity, facial obstruction factors, and adverse lighting conditions, this system eliminates these shortcomings.

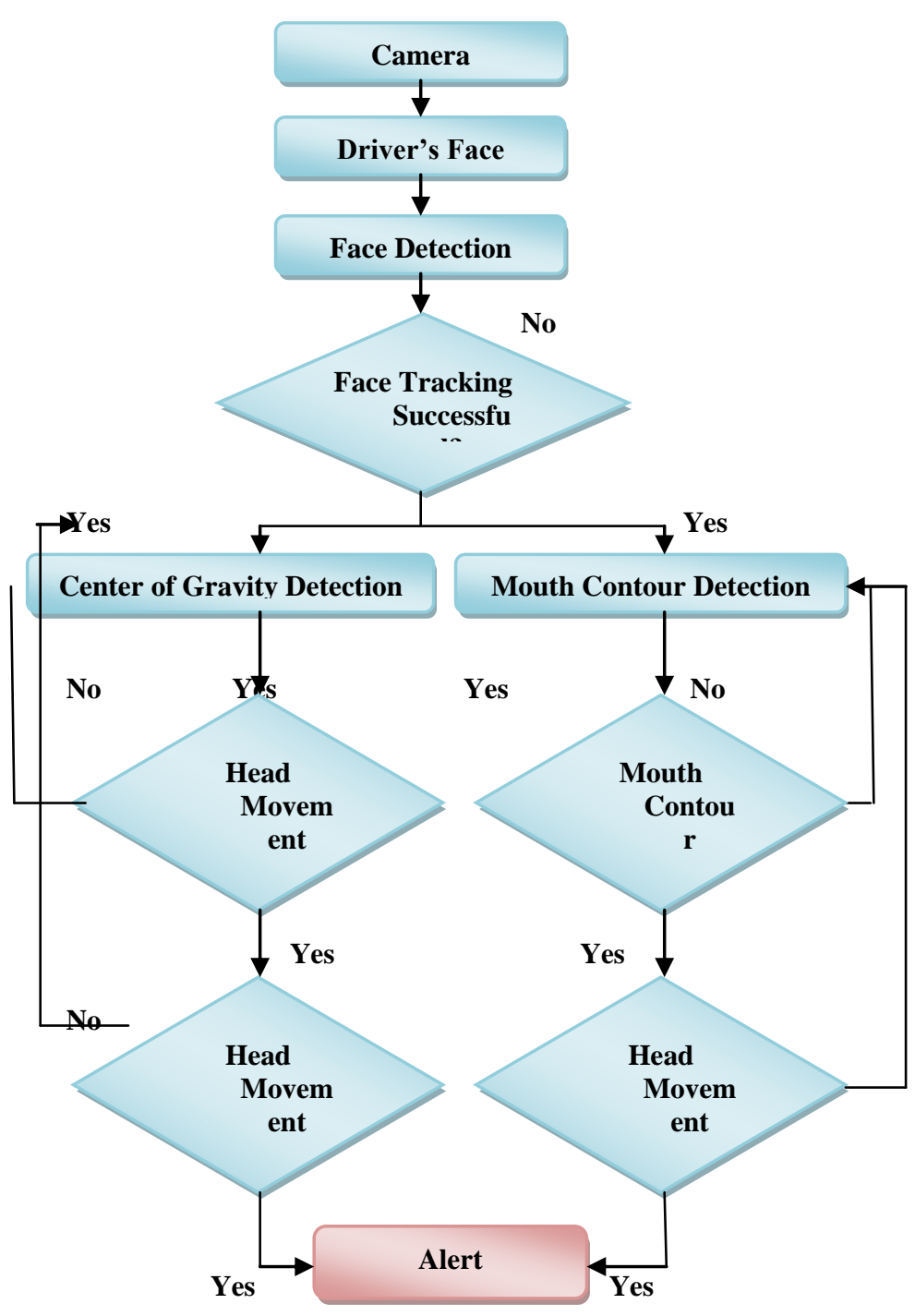

Figure 1. System Flow

In this approach, the driver's face is continuously captured using a video camera that is installed under the front mirror inside the car. Next, detecting drowsiness involves three main steps to measure changes in facial gestures that imply drowsiness. First, the driver's face is detected and tracked in 
the series of frame shots taken by the camera. Second, after detection of the face, head movement is detected. Third, the yawning state is detected. Figure 1 demonstrates several steps of the algorithm.

\section{RELATED WORK}

As driver's drowsiness is one of the major causes of road accidents, it is well studied and addressed in much research work. This increases transportation safety and decrease the number of deaths caused by fatigued drivers. Different body gestures of driver were used to measure drowsiness for which the methods for assessing measurement of the driver's state, driver performance and a combination of the driver's state and performance were used.

Primarily techniques based on computer vision were used [2].Tuncer et al. proposed an assistant system to track a lane for those drivers who are not able to perform a good job of lane keeping [3]. With this method required steering actions were calculated and sent to vehicle model. Driver's assessment was determined in the context of a road departure warning and intervention system [4]. The position of vehicle was used as input and performance was determined by analyzing the changes in the bandwidth or parameters of such a model.

Fatigue detection method based on brain and visual activity was proposed by [5]. Another method assessed fatigue by using electrocardiography, eye movement [6]. A pulse wave sensor was used to detect drowsiness [7]. Hayashi et al. proposed another method of driver drowsiness detection by pulse wave analysis with a neural network [8].

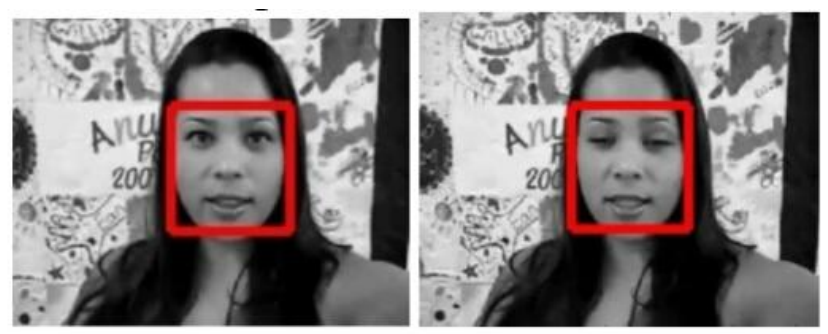

Figure 2. Face Detection

A clustered background based on segmentation was used by Sirohey in order to identify the face [9] as shown in Figure 2. [10] developed a new method based on locating facial gestures. A method of face detection based on a morphology technique to perform eye analogue segmentation was proposed by [11]. [12] introduced a driver drowsiness monitoring system based on a combination of eyes and mouth gestures. The proposed system determines the state of mouth and eyes by analyzing their feature points using Back Propagation neural networks in order to check for

conditions that involve driver drowsiness. A face detection system that uses histogram intersection in the HSV color space to highlight the skin region was proposed by [13]. [14] proposed the following method: the frontal view face is detected based on template matching. The extracted edges from Sobel filtering will be grouped together to locate the face. Then, the same procedure will be repeated to find other facial features such as eyes, mouth and nose in the candidate face. Face detection based on BP neural network and Bayesian decision was described by Liu et al [15]. The Bayesian decision theory was used to classify the face or nonface pattern and also to improve the correct rate of face detection. [16] took advantage of grey projection and Gabor wavelets to detect the mouth corners and uses LDA to find a linear combination of the those features to detect the yawning mouth. [17] detects the face using Viola-Jones technique and extracts the mouth region, in which lips were searched for through spatial fuzzy c-means (s-FCM) clustering. [18] proposed the use of mouth geometrical features to detect yawning. [19] used thecascade of classifier as proposed by Viola-Jones face detectionapproach. It then used a support vector machine to train theclassifier with the mouth features in yawning condition. Another approach to facial movement analysis wasproposed in [20] by using Adaboost and multinomial ridgeregression to train the classifier of different facial actions suchas blinking and yawn motions.

[21] proposed a method of hypo-vigilance detection by processing of the eye region and without an explicit eye detection stage. A drowsiness detection method based on eyelid movement was proposed by [22]. [23] used a method of fatigue detection by applying the Structural Similarity Measure to find the eye location.

The method to eliminate the frame region in the face area to reduce the search area was described by [24]. [25] required the use of two cameras: a low resolution camera for the face and a high resolution one for the mouth. It uses haar-like features to detect driver's mouth, and yawning is detected based on the aspect ratio of the mouth. Two cameras are used in the proposed method of $\mathrm{Li}$ et al. [26] to detect driver's fatigue in real time. In their method, one low resolution camera was installed in the car to supply the driver's head position and one high resolution camera to locate the mouth region in each frame. In the method studied by Fan et al. [27], the driver's face was detected by using a Gravity-Center template. Jimenez et al. [28] described a new method of fatigue detection in drivers based on the percentage of closing eyes and detection of yawning and nodding.

Given a single image, the goal of face detection is to identifyall image regions that contain a face regardless of its position,orientation, and lighting conditions. Such a problem is challenging because faces are nonrigid and have a high degree of variability in size, shape, color, and texture. The orientation of the face can also be a challenge in the detection process. The other important factor is lighting conditions. This is mainly affected by the environment light that can change depending on the time of the day and weather conditions. [29] addresses the problem of face detection in the presence of illumination variations and proposes a solution based on the use of an adaptive illumination normalization procedure. Also use of infrared cameras is incompatible due to economic reasons. In appearance based methods, the face models are learned from a set of training images, which include the representative variability of facial appearance. Such methods can take advantage of Neural Networks. However, when it comes to a yawning face, it sometime fails to detect the whole face down to the bottom of the chin especially when the mouth is wide open. The presence of beards, mustache, glasses, and sunglasses create a great deal of challenge. Sunglasses in particular make it difficult as they are commonly used during driving and not only cover a considerable area of the face but also cause a shadow on it, rendering many of the existing detection technologies ineffective. Hence despite considerable research in this area, today only a few expensive yawning monitoring systems exist. There are three main reasons why existing techniques, such as the ones described above, are not robust enough for a production-grade and serious consumer system. 
Proposed approach aims at eliminating yawning detection's dependency on the previously mentioned factors such as facial occlusion and lighting change. It also includes the use of more advanced algorithms for face detection leading to a higher efficiency and robustness of the system.

\section{PROPOSED SYSTEM}

To detect the drowsiness of drivers, the most important element is a reliable system to monitor the driver and determine whether he/she is drowsy Even though drowsiness is a concept that is understood by anyone, it is very complex task to quantify it. This section proposes two implemented methods of drowsiness monitoring system based on yawning detection and head movement detection using face detection in order to reach the goal of having a reliable and robust system. In this system the day - night camera will be placed in front of driver's seat on dashboard. That camera keeps continuous watch on driver's behavior and gestures and catches continuous images in video feed. This video feed will be dividedinto number of frames.
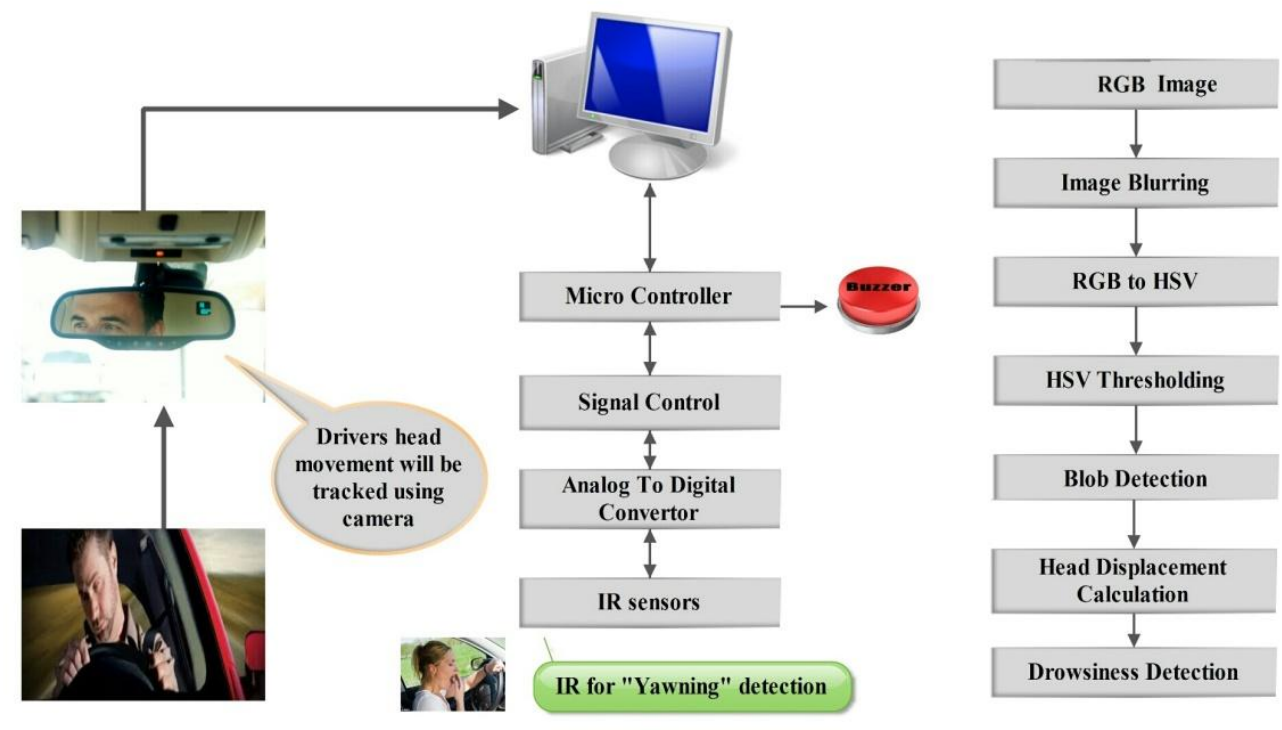

Figure 3. Proposed System

After this face detection will be carried out with the help of RGB color space model. However, it should be noted that skin segmentation in RGB color space is quite sensitive to illumination variation. Therefore to increase the detection efficiency HSV color model is added. Once Face is detected further two operations are performed:

\section{Calculating center of gravity of face.}

\section{Mouth contour detection.}

When center of gravity of face is calculated it is able to track the head movement, once head movement is detected it will provide an alert mechanism. On the other hand after mouth contour detection if yawn is detected an alert mechanism is provided.

\section{APPLICATIONS}

The proposed system can be used for variety of applications. One of them is heavy duty vehicles for example trucks. Also it can be used for commercial vehicles. Another application is passenger vehicles. Many people use public transport facility for travelling. For their safety this system can be used. Cranes are mainly used for lifting heavy things and transporting them to other places. In such cases drowsiness of driver may cause serious problems. So for overload cranes and mobile cranes this system can be used.

\section{FUTURE SCOPE}

1. Real time detected data of driver's drowsiness can be stored on the cloud system to monitor driver's performance.
2. LAB/Edge Detection Algorithm can be used for Face Detection.

3. Rather using alarm Automatic Braking System can be used which will reduce the speed of the vehicle.

4. The vehicle can be automatically parked by first using Automatic braking system, which will reduce the speed and concurrently will turn on the parking lights of the vehicles

5. Using Pressure sensor on the steering alarm Automatic braking System can be set in case of drowsiness.

6. By using wireless Technology if the driver gets drowsy an alert message can be sent to a selected person's mobile by using GSM module along with the alarm in vehicle.

\section{REFERENCES}

[1] S.G. Klauer , T. A. Dingus, Neale , V. L. , Sudweeks , J.D. , and Ramsey, DJ, ”The Impact of Driver Inattention on Near- Crash/Crash Risk: An Analysis Using the 100Car Naturalistic Driving Study Data," Virginia Tech Transportation Institute, Technical Report \# DOT HS 810594.

[2] W. Qiong, Y. Jingyu, R. Mingwu and Z. Yujie, "Driver Fatigue Detection: A Survey," The Sixth World Congress on Intelligent Control and Automation, vol. 2, pp. $8587-$ $8591,2006$.

[3] Tuner, L. ven , F. Co kun and E. Karsl gil, Vision based lane keeping assistance control triggered by a driver 
inattention monitor," in IEEE International Conference on Systems Man and Cybernetics (SMC), Istanbul, 10-13 Oct. 2010

[4] T. Pilutti and A. Ulsoy, "Identification of driver state for lane-keeping tasks," IEEE Transactions on Systems, Man and Cybernetics, Part A: Systems and Humans, vol. 29, no. 5, pp. 486-502, 1999.

[5] A. Picot, S. Charbonnier and A. Caplier, "On-Line Detection of Drowsiness Using Brain and Visual Information," IEEE Transactions on Systems, Man and Cybernetics, Part A: Systems and Humans, no. 99, pp. 112, 2011.

[6] G. Furman, A. Baharav, C. Cahan and S. Akselrod, "Early detection of falling asleep at the wheel: A Heart Rate Variability approach," Computers in Cardiology, pp. 1109-1112, 2008.

[7] $\mathrm{S}$. Hu and R. Bowlds, "Pulse wave sensor for nonintrusive driver's drowsiness detection," in Engineering in Medicine and Biology Society, 2009. EMBC 2009. Annual International Conference of the IEEE, Minneapolis, MN, 2009.

[8] K. Hayashi, K. Ishihara, H. Hashimoto and K. Oguri, "Individualized drowsiness detection during driving by pulse wave analysis with neural network," in Proceedings of the 8th International IEEE Conference on Intelligent Transportation Systems, Austria , 2005.

[9] S. Sirohey, "Human Face Segmentation and Identification," in Technical Report CS-TR-3176, Maryland, 1993.

[10] H. Graf, T. Chen, E. Petajan and E. Cosatto, "Locating Faces and Facial Parts," in Proc. First Int'l Workshop Automatic Face and Gesture Recogniction, 1995.

[11] C. Han, H. Liao, K. Yu and L. Chen, "Fast Face Detection via Morphology-Based Pre-Processing," in Proc. Ninth Int'l Conf. Image Analysis and Processing, 1998.

[12] Y. Ying, S. Jing and Z. Wei, "The Monitoring Method of Driver's Fatigue Based on neural network," in International Conference on Mechatronics and Automation, 2007, Harbin, 2007.

[13] D. Saxe and R. Foulds, "Towards Robust Skin Identification in Video Images," in Proc. Second Int'l Conf. Automatic Face and Gesture Recognition, 1996.

[14] I. Craw, H. Ellis and J. Lishman, "Automatic Extraction of Face Features," Pattern Recognition Letters, vol. 5, pp. 183-187, 1987.

[15] X. Liu, G. Geng and X. Wang, "Automatically face detection based on BP neural network and Bayesian decision," in Sixth International Conference on Natural Computation (ICNC), Yantai, Shandong, 2010.

[16] X. Fan, B. Yin, Y. Fun. "Yawning Detection For Monitoring Driver Fatigue." In: Proc. Sixth International
Conf. on Machine Learning and Cybernetics, Hong Kong, 2007, pp. 664-668.

[17] T. Azim, M.A. Jaffar, A.M. Mirza. "Automatic Fatigue Detection of Drivers through Pupil Detection and Yawning Analysis." In: Proc. Fourth International Conf. on Innovative Computing, Information and Control, 2009, pp. 441-445.

[18] T.Wang, P. Shi. "Yawning Detection For Determining Driver Drowsiness." IEEE International Workshop VLSI Design \& Video Tech. Suzhou

[19] M. Saradadevi and P. Bajaj, "Driver Fatigue Detection Using Mouth and Yawning Analysis," IJCSNS International Journal of Computer Science and Network Security, vol. 8, no. 6, pp. 183-188, June 2008.

[20] E. Vural, M. Cetin, A. Ercil, G. Littlewort , M. Barlett and J. Movellan, "Drowsy Driver Detection Through Facial Movement Analysis".

[21] M. Sigari, "Driver Hypo-vigilance Detection Based on Eyelid Behavior," in Seventh International Conference on Advances in Pattern Recognition, 2009.

[22] D. Liu, P. Sun, Y. Xiao and Y. Yin, "Drowsiness Detection Based on Eyelid Movement," in 2010 Second International Workshop on Education Technology and Computer Science (ETCS), 2010

[23] M. Omidyeganeh, A. Javadtalab and S. Shirmohammadi, "Intelligent driver drowsiness detection through fusion of yawning and eye closure," in IEEE International Conference on Virtual Environments Human-Computer Interfaces and Measurement Systems (VECIMS), 2011.

[24] P. Viola and M. Jones, "Robust real-time face detection," International Journal of Computer Vision , vol. 57, no. 2, pp. 137-154, 2001

[25] L. Li, Y. Chen , Z. Li. "Yawning Detection for Monitoring Driver Fatigue Based on Two Cameras." In: Proc. 12th International IEEE Conf. on Intelligent Transportation Systems, St. Louis, MO, USA, 2009, pp. $12-17$

[26] L. Li, Z. Li and Y. Chen, "Yawning detection for monitoring driver fatigue based on two cameras," in 12th International IEEE Conference on Intelligent Transportation Systems, 2009

[27] X. Fan, B. Yin and Y. Sun, "Yawning Detection for Monitoring Driver Fatigue," in International Conference on Machine Learning and Cybernetics, 2007.

[28] R. Jimenez, F. Prieto and V. Grisales, "Detection of the Tiredness Level of Drivers Using Machine Vision Techniques", Electronics, Robotics and Automotive Mechanics Conference, 2011.

[29] H. Selleahewa, S. Jassim, "Image-Quality-Based Adaptive Face Recognition ", IEEE Transaction on vol 59 , no.4. 